\title{
Astroglial NF-kB contributes to white matter damage and cognitive impairment in a mouse model of vascular dementia
}

\author{
Raman Saggu1', Toni Schumacher ${ }^{1}$, Florian Gerich', Cordula Rakers', Khalid Tai ${ }^{1}$, Andrea Delekate ${ }^{1}$
} and Gabor C. Petzold ${ }^{1,2^{*}}$

\begin{abstract}
Vascular cognitive impairment is the second most common form of dementia. The pathogenic pathways leading to vascular cognitive impairment remain unclear but clinical and experimental data have shown that chronic reactive astrogliosis occurs within white matter lesions, indicating that a sustained pro-inflammatory environment affecting the white matter may contribute towards disease progression. To model vascular cognitive impairment, we induced prolonged mild cerebral hypoperfusion in mice by bilateral common carotid artery stenosis. This chronic hypoperfusion resulted in reactive gliosis of astrocytes and microglia within white matter tracts, demyelination and axonal degeneration, consecutive spatial memory deficits, and loss of white matter integrity, as measured by ultra high-field magnetic resonance diffusion tensor imaging. White matter astrogliosis was accompanied by activation of the pro-inflammatory transcription factor nuclear factor (NF)-kB in reactive astrocytes. Using mice expressing a dominant negative inhibitor of NF-kB under the control of the astrocyte-specific glial fibrillary acid protein (GFAP) promoter (GFAP-IkBa-dn), we found that transgenic inhibition of astroglial NF-kB signaling ameliorated gliosis and axonal loss, maintained white matter structural integrity, and preserved memory function. Collectively, our results imply that pro-inflammatory changes in white matter astrocytes may represent an important detrimental component in the pathogenesis of vascular cognitive impairment, and that targeting these pathways may lead to novel therapeutic strategies.
\end{abstract}

Keywords: Astrocytes, vascular cognitive impairment, Glia, NF-kB, Diffusion tensor imaging (DTI), Neuroinflammation

\section{Introduction}

Vascular cognitive impairment (VCI) is a common neurodegenerative disease characterised by impaired cognitive function attributable to cerebrovascular pathology [1]. In its pure form, $\mathrm{VCI}$ is the second most common form of dementia and represents an important co-factor for the development of Alzheimer's disease and other neurodegenerative disorders [2]. Neuropathologically, $\mathrm{VCI}$ is characterised by demyelination and axonal loss within white matter tracts [3], which has traditionally been attributed to the direct effects of chronic oxygen deprivation. However, several lines of evidence suggest

\footnotetext{
* Correspondence: gabor.petzold@dzne.de

${ }^{1}$ German Center for Neurodegenerative Diseases (DZNE), Ludwig-Erhard-Allee 2, 53175 Bonn, Germany

${ }^{2}$ Department of Neurology, University Hospital Bonn, Sigmund-Freud-Str. 25, 53127 Bonn, Germany
}

that chronic neuroinflammation may also play a detrimental role in the initiation and progression of VCI. First, cerebrovascular and cardiovascular diseases, which are risk factors for $\mathrm{VCI}$, induce a chronic pro-inflammatory environment, particularly in and around blood vessels $[4,5]$. Second, increased levels of pro-inflammatory markers have been detected within white matter lesions in animal models and post-mortem studies of VCI patients $[6,7]$, and alterations of blood-brain barrier integrity are a central and perhaps aggravating element of VCI $[8,9]$. Finally, in patients and animal models of VCI, reactive gliosis of microglia and astrocytes is present within the white matter $[10,11]$. Reactive astrogliosis is a pervasive defence mechanism in response to cerebral injury that may promote neurodegeneration or neuroprotection in a context-dependent manner [12]. However, the roles of astrogliosis and the various inflammatory 
cascades mediated by reactive astrocytes in chronic cerebral hypoperfusion and VCI remain poorly understood.

Here, we have used an animal model that recapitulates many of the neuropathological and behavioural hallmarks of VCI [13]. We demonstrate that reactive astrogliosis induces activation of the pro-inflammatory nuclear factor (NF)-kB pathway in astrocytes, and that transgenic inhibition of this pathway confers protection against axonal loss, helps to promote white matter integrity, and preserves cognitive function.

\section{Materials and methods}

\section{Animals}

All experiments were approved by a local animal welfare committee. We used 10-14 week-old male GFAP-IkB $\alpha$ $\mathrm{dn}$ mice [14] and their wildtype age-matched male littermates. Animals were housed in groups (two to five per cage) on a $12 \mathrm{~h}$ light/dark cycle with food and water available ad libitum.

\section{Chronic hypoperfusion model}

Gold plated stainless steel microcoils (Sawane) were wrapped around both common carotid arteries in isoflurane-anaesthetised mice [13]. Informed by earlier studies that revealed either subtle $[15,16]$ or very pronounced changes [17], we modified the existing model by using custom-made coils with an internal diameter of $170 \mu \mathrm{m}$. Animals were anaesthetised with isoflurane $(1.5 \% \mathrm{v} / \mathrm{v})$ and maintained on a heating pad $\left(37^{\circ} \mathrm{C}\right)$. The common carotid arteries were exposed through a midline cervical incision, and the microcoils were wrapped around both common carotid arteries. A 30 min interval was allowed between each microcoil insertion. Sham-treated animals underwent identical procedures except that coils were not implanted. Local lidocaine gel and intraperitoneal buprenorphine were applied as analgesics. Mice from each litter were randomised to either microcoil insertion or sham surgery. All experiments and subsequent data analysis were conducted in a blinded manner, with respect to genotype and treatment. Mice were excluded if visual damage to the carotid arteries or bleeding during surgery occurred. In addition, carotid arteries were inspected for patency at the end of experiments, and animals with occluded carotid arteries were also excluded.

\section{Laser speckle perfusion imaging and PcomA assessment}

Animals were anaesthetised with isoflurane $(1.5 \% \mathrm{v} / \mathrm{v})$, and the skull was exposed through a midline incision. A cranial window was created and closed by gluing a glass coverslip onto the skull and adhering the skin to the coverslip rim. For imaging, mice were anaesthetised with isoflurane $(1 \% \mathrm{v} / \mathrm{v})$ and fixed within a stereotaxic frame (Narishige). Brain perfusion was determined through a closed cranial window in anaesthetised mice using PeriCam PSI Laser Speckle Imager and PIMsoft software (Perimed). PcomA development was determined by transcardial perfusion with India ink (Pelikan; $10 \%$ in water containing $10 \%$ gelatine), followed by decapitation and fixation in $4 \%$ paraformaldehype (PFA). PcomA was scored as: 0 , absent; 1 , hypoplastic; 2 , truncal. A single PcomA score was calculated by averaging left and right scores.

\section{Ultra high-field MRI}

MRI of isoflurane-anaesthetised mice was performed on an $11.7 \mathrm{~T}$ horizontal small-bore magnet (Biospec 117/16, Bruker) using a two-element transmit/receive proton $\left({ }^{1} \mathrm{H}\right)$ cryocoil (Bruker Biospin). Body temperature was maintained at $37{ }^{\circ} \mathrm{C}$ via an integrated water heating system. Respiratory rate was maintained at 70-80 cycles $/ \mathrm{min}$. Anatomical images were acquired using a rapid acquisition relaxation enhancement (RARE) $\mathrm{T}_{2}$-weighted $\left(\mathrm{T}_{2}-\mathrm{w}\right)$ sequence [echo time $(\mathrm{TE})=0.01 \mathrm{~s}$; repetition time $(\mathrm{TR})=2.5 \mathrm{~s}$; in-plane resolution $45 \mu \mathrm{m}^{2}$ ], and a RARE $T_{1}$-weighted $\left(T_{1}-\mathrm{w}\right)$ sequence $(T E / T R=0.01 \mathrm{~s} / 1 \mathrm{~s}$; in-plane resolution $45 \mu \mathrm{m}^{2}$ ). White matter integrity was assessed using an EPI DTI sequence (TE/TR 26/4000 ms; $b$ value $1000 \mathrm{~s} / \mathrm{mm}^{2} ; 60$ diffusion directions; 5 b0 images; in-plane resolution $100 \mu \mathrm{m}^{2}$ ).

\section{Immunohistochemistry}

Coronal sections $(20 \mu \mathrm{m})$ were obtained from PFA-fixed hemispheres, blocked with $10 \%$ normal goat serum (Vector Labs) and 0.3 \% Triton-X100 (Sigma) in PBS for $1 \mathrm{~h}$, and incubated with primary antibodies in PBS containing $5 \%$ goat serum and $0.05 \%$ Triton overnight at $4{ }^{\circ} \mathrm{C}$. The following primary antibodies were used: rabbit anti-GFAP (1:1000; Z0334, Dako), rat anti-GFAP (1:1000; 13-0300, Invitrogen), rabbit anti-Iba1 (1:500; \#019-19741, Wako), mouse anti-SMI312 (1:500, smi-312r, Covance), rabbit anti-p65 (1:100; sc-372, Santa Cruz; or \#3033, Cell Signaling). Stainings were visualised using secondary antibodies from goat conjugated with Alexa Fluor 488, 596 or 647 (1:1000; Invitrogen; $2 \mathrm{~h}$ at room temperature). Nuclei were stained with Hoechst 33258 (1:1000; Invitrogen). Omission of primary antibodies served as negative controls. Confocal images were acquired using a confocal laser scanning microscope (LSM 700; Zeiss).

\section{Cytokine and NF-kB quantification}

Mice were sacrificed, and the corpus callosum was dissected and stored in liquid nitrogen. Samples were homogenised in PBS containing protease/phosphatase inhibitor (Thermo Scientific) using a Precellys 24 homogeniser (Peqlab), and lysed in RIPA buffer (25 mM TRIS, $150 \mathrm{mM}$ $\mathrm{NaCl}, 1$ \% NP-40, 0.5 \% Na-deoxycholate, $0.1 \%$ SDS, $\mathrm{pH}$ 7.2). Quantitative cytokine determination was 
performed using an electrochemiluminescence ELISA (Mouse ProInflammatory Ultra-Sensitive Kit, Meso Scale Discovery). Signals were measured on a SECTOR Imager 2400 reader (Meso Scale Discovery). For NF-kB measurements, nuclear extracts were created from whole brain homogenates (Nuclear Extraction Kit, \#2900, Millipore), and NF-kB activity in nuclear extracts was determined with a commercial assay (EZ-TFA assay, \#70-610, Millipore) according to the manufacturer's instructions using a microplate reader (FLUOstar Omega, BMG Labtech). All measurements were performed in duplicates.

\section{Behavioural tests}

Male GFAP-IkB $\alpha$-dn and wildtype mice have similar phenotypes in behavioural paradigms, including spatial learning and memory [18]. All tests were recorded and analysed using Ethovision XT9 (Noldus). The Y maze consisted of three arms (length, $34.5 \mathrm{~cm}$; width, $8 \mathrm{~cm}$; height, $12.5 \mathrm{~cm}$ ) extruding at equal angles from a central platform. The Y Maze test was modified from previous designs [19]. During the acquisition trial, one arm was closed and mice were placed at the end of an open arm (chosen at random) and allowed to explore the two open arms for $5 \mathrm{~min}$. After $24 \mathrm{~h}$, mice were returned to the maze with all 3 arms open; the start point corresponding to the individual start point of each mouse in the acquisition trial, and allowed to explore for $5 \mathrm{~min}$. Arm preference was quantified as the ratio between the time spent in one arm and the time spent in all arms (preferency index). For open field exploration, mice were placed in the centre of a dimly lit (20-30 lx) arena. The area was virtually divided into a centre and an area $\leq 5 \mathrm{~cm}$ to the walls (surround). General locomotor activity was quantified as total distance moved and velocity. The level of anxiety was estimated by the percentage of time spent in the surround.

\section{Data analysis}

Immunohistochemical images were imported into ImageJ 1.50 (W. Rasband, NIH), smoothed and despeckled using a median filter, and converted to an 8-bit grey level image. Following contrast enhancement (0.4\% saturated pixels), images were binarised with a threshold defined as the mean background intensity plus the standard deviation of background intensity multiplied by 3 . Astrocyte area coverage and diameter, and micoglial cell count were determined automatically by ImageJ. For astrocyte process length, binarised images were skeletonised and analysed using the Analyze Skeleton (2D/3D) plugin for ImageJ. These semi-automatic quantifications were compared with a subset of data ( $n=3$ from each group) that was quantified by manual analysis; no significant difference was detected between the analysis methods (data not shown). GFAP-positive astrocytes were considered positive for p65 when they showed a spherical somatic p65 staining that was smaller than the GFAP-positive cell soma and overlapped with the Hoechst 33258 nuclear counterstain. Mean pixel intensity of SMI312 staining in 8-bit grey level images was taken as a measure of axonal integrity. Demyelination was analysed in LFB-stained sections using a semi-quantitative scoring system $(0=$ no demyelination; 1 = visible fiber disarrangement; 2 = vacuoles and focal demyelination; $3=$ widespread/continuous demyelination). Examples are provided in Additional file 1: Figure S1. Analysis of the corpus callosum was performed within pre-defined areas $(0.75 \mathrm{~mm}$ and $2.0 \mathrm{~mm}$ from the midline). All measurements were taken in triplicates, and the values averaged. Laser speckle contrast data were down-sampled, exported into Excel (Microsoft), and normalised to perfusion levels before microcoil implantation. Paravision 5.1 (Bruker) was used to generate the parametric images used for white matter analysis. Fractional anisotropy (FA), apparent diffusion coefficient (ADC), axial diffusivity $(\mathrm{AD})$ and radial diffusivity (RD) were measured. Direction-encoded colour (DEC) maps based on the primary eigenvectors were generated using inhouse software. The colour determines the principal direction (X, Y or Z) of the primary eigenvector exhibiting the largest diffusion coefficient from the measured diffusion tensor: red designates medial to lateral orientation, blue represents dorsal to ventral orientation and green exhibits the rostral to caudal orientation. Regions of interest (ROIs) including the corpus callosum, external capsule and internal capsule were manually drawn onto the diffusion tensor images, and verified by comparison with the corresponding $\mathrm{T}_{2}$ - $\mathrm{W}$ image and a stereotaxic mouse brain atlas [20]. Heatmaps were generated by a) registering the FA images to a reference image within the respective cohort and b) setting landmarks on trace-weighted diffusion images and transferring them onto their respective FA images. Registration was performed using the bUnwarp plugin (version 2.6.3) for ImageJ.

\section{Statistical analysis}

Comparisons between two groups were conducted using the Mann-Whitney test. Differences between several groups were analysed using the Kruskal-Wallis test followed by Dunn's multiple comparisons test. Data were analysed using Prism 6 (GraphPad), and are expressed as mean \pm SEM; $p<0.05$ was accepted as statistically significant.

\section{Results}

Gliosis, demyelination, axonal loss and memory deficits in a mouse model of $\mathrm{VCl}$

We modified a model of bilateral common carotid artery stenosis (BCAS) [13] by placing microcoils around both common carotid arteries to induce chronic cerebral hypoperfusion in mice (Fig. 1a). Longitudinal laser speckle 

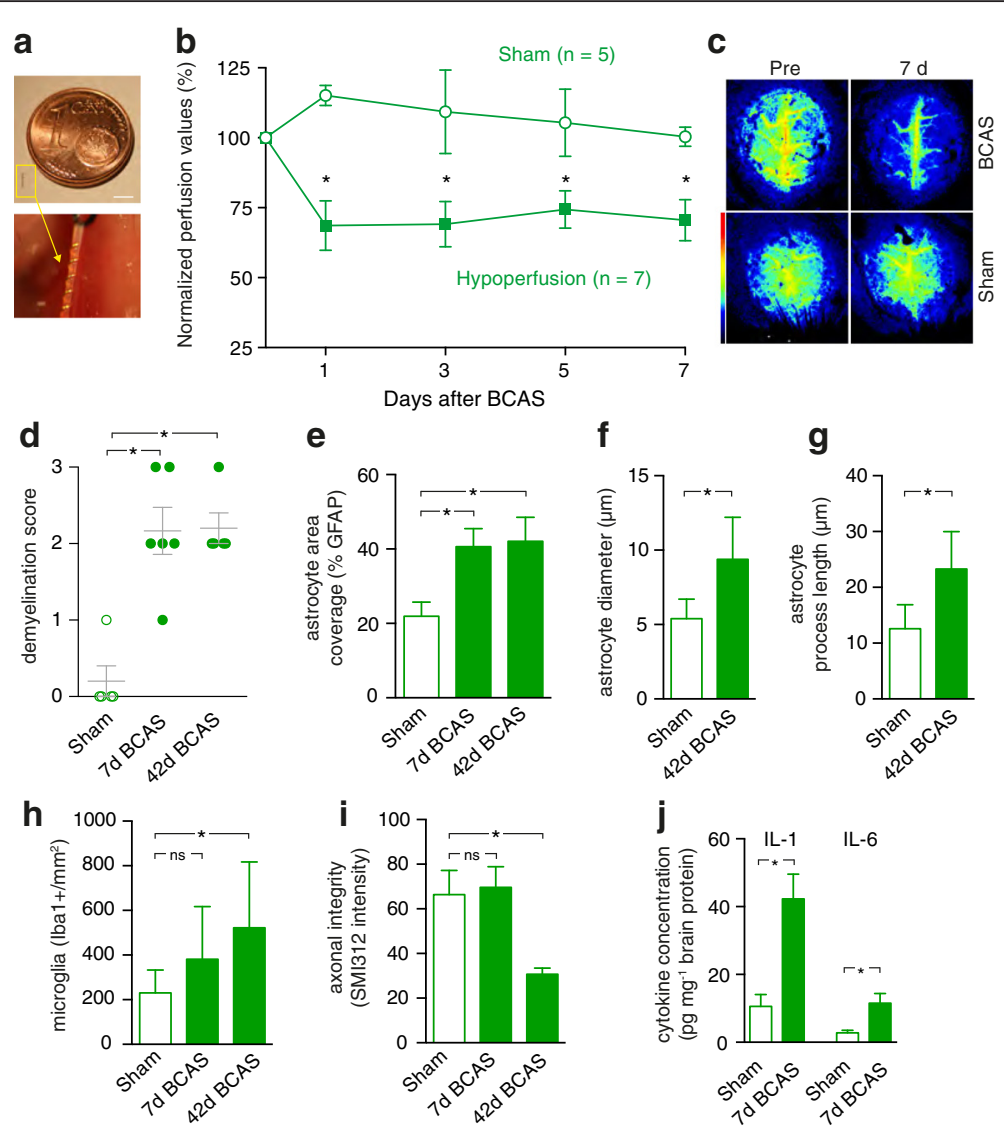

Fig. 1 Chronic hyperperfusion induces demyelination, gliosis, axonal deterioration, and cognitive impairment. a Mice were subjected to bilateral carotid artery stenosis (BCAS) by wrapping gold-plated microcoils (internal diameter, $170 \mu \mathrm{m}$ ) around the common carotid arteries. Scale bar, $3 \mathrm{~mm}$. b Laser speckle contrast imaging of anaesthetised mice revealed long-lasting cerebral hypoperfusion after BCAS compared to shamtreated mice ( $p<0.05$, Mann-Whitney test). c Representative examples of laser speckle contrast measurements of cerebral blood flow through chronic cranial windows in mice subjected to BCAS or sham surgery before (Pre) and $7 \mathrm{~d}$ after the intervention. The look-up table represents arbitrary perfusion units, indicating reduced blood flow after BCAS. $\mathbf{d}$ Luxol Fast Blue staining of the corpus callosum $(0=$ no demyelination; 1 = visible fiber disarrangement; 2 =vacuoles and focal demyelination; 3 = widespread/continuous demyelination) revealed demyelination $7 \mathrm{~d}$ and $42 \mathrm{~d}$ after BCAS ( $n=6$ for each group; $p<0.05$, Kruskal-Wallis test followed by Dunn's multiple comparisons test). e- $\mathbf{g}$ The area covered by GFAP-positive astrocytes, astrocyte diameter and astrocyte process length were increased in mice subjected to BCAS in the corpus callosum, indicating reactive astrogliosis ( $n=6$ for each group; $p<0.05$, Mann-Whitney test). $\mathbf{h}$ Mild reactive microgliosis was also evident 6 weeks after BCAS, indicated by an increase in Iba1-positive cells ( $n=6$ for each group; $p<0.05$, Mann-Whitney test). i In parallel, there was a decrease in the density of SMI312-positive axons in the corpus callosum after 6 weeks, indicating axonal loss $(n=6$ for each group; $p<0.05$, Mann-Whitney test). $\mathbf{j}$ Electrochemiluminescence measurements revealed an increase in the pro-inflammatory cytokines IL-1 and IL-6 in homogenates from the corpus callosum ( $n=8$ for each group; $p<0.05$, Mann-Whitney test)

imaging demonstrated a reduction in cerebral blood flow by $32 \pm 9 \% 24 \mathrm{~h}$ and $29 \pm 8 \% 7$ days following BCAS induction, respectively, whereas blood flow remained unaltered in sham-treated mice (Fig. 1b-c). Separate cohorts of mice, perfused either 1 week or 6 weeks after BCAS induction, exhibited white matter demyelination (Fig. 1d), reactive astrogliosis (Fig. 1e-g), microglial activation (Fig. 1h), and delayed degradation of white matter axonal integrity (Fig. 1i). Reactive astrogliosis was evident by increased area coverage, cell size and process length, although the latter parameter is also influenced by GFAP redistribution induced by astrogliosis [12]. The number of GFAP-positive astrocytes in the corpus callosum did not differ between the groups, owing to the high fraction of GFAP-positive astrocytes already present in the white matter $\left(148 \pm 12 / \mathrm{mm}^{2}\right.$ and $159 \pm 10 / \mathrm{mm}^{2}$, respectively; $n=6$ for each group; $p>0.05$, Mann-Whitney test). No difference was seen in the number of NeuNpositive neuronal cell bodies in the cortex (Sham, $1987 \pm$ $211 / \mathrm{mm}^{2}$; BCAS, $2027 \pm 274 ; n=6$ for each group; $p>0.05$, Mann-Whitney test). There was no change in the rostro-caudal diameter of the corpus callosum, measured in coronal sections, in mice subjected to BCAS compared with sham-treated mice $(211 \pm 26 \mu \mathrm{m}$ vs. $201 \pm 32 \mu \mathrm{m} ; n=6$ for each group; $p>0.05$, MannWhitney test). Representative examples are shown in the 
Additional file 2: Figure S2. Changes comparable to those observed in the corpus callosum were detected in the internal capsule and external capsule (Additional file 3: Figure S3).

Moreover, we found that the concentrations of the proinflammatory cytokines, interleukin-1 and interleukin-6, were increased in homogenates of the corpus callosum isolated from mice subjected to BCAS (Fig. 1j).

To investigate spatial long-term memory, we used a two-trial Y maze spatial memory task [19]. In the first trial (acquisition), mice were allowed to visit two arms of the maze, while the third arm was blocked. In the second trial (retrieval) performed $24 \mathrm{~h}$ later, mice had access to all three arms. As expected, given the natural tendency of rodents to explore new environments, sham-treated mice showed a strong preference for the novel arm in the retrieval trial (Fig. 2a-b). By contrast, mice subjected to BCAS showed no preference for the novel arm (Fig. 2b), indicating decreased spatial memory function. Spontaneous locomotor activity and the level of anxiety, which were investigated in the open field test, did not differ between the groups (Fig. 2c-e) indicating that the deficits of hypoperfused mice in the $\mathrm{Y}$ maze test were due to impaired memory function and not to a decline in motor function or general behavioural alterations.

\section{NF-kB is activated by chronic hypoperfusion in reactive} white matter astrocytes

One consequence of the expression of pro-inflammatory cytokines such as IL-1 is the activation of the nuclear factor (NF)-kB signaling cascade in reactive astrocytes [21]. Under control conditions, inactive NF-kB is localised to the cytoplasm by its interaction with inhibitory $\mathrm{IkB}$ proteins such as $\mathrm{IkB} \alpha$. Dissociation from $\mathrm{IkB}$ and subsequent nuclear translocation of NF- $k B$ is initiated by the degradation of IkB through cytokine-induced IkB kinase activation [22]. Once activated, NF-kB acts as an important regulator of reactive gliosis, inflammation, myelination and neuronal survival in different disease models [12, 14, 23].

Therefore, we investigated the occurrence of activated nuclear NF-kB by immunohistochemistry against the active p65 (RelA) subunit of NF-kB. We found that $27.2 \pm$ $7.1 \%$ of all GFAP-positive white matter reactive astrocytes in hypoperfused mice were positive for nuclear (i.e. active) p65 ( $n=7$ mice; Fig. 3a). By contrast, only sparse and cytosolic (i.e. inactive) p65 labeling was visible in astrocytes from sham-treated mice $(3.8 \pm 1.8 \%, n=6$ mice; Fig. 3b). White matter microglia and neurons were only very rarely positive for nuclear p65 in chronic hypoxic mice $(3.4 \pm 0.9 \%$ of Iba1-positive microglia and $0.8 \pm$
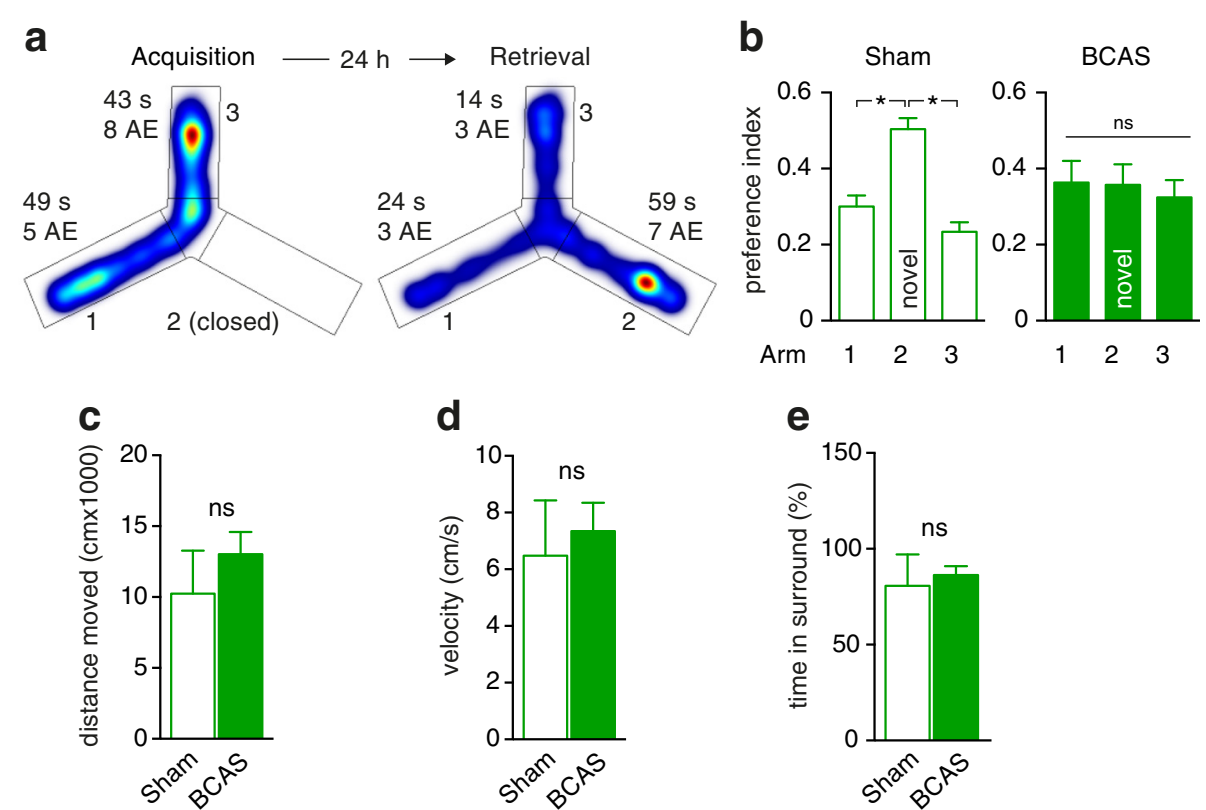

Fig. 2 Chronic hypoperfusion induces a decline in spatial long-term memory. a Representative example of spatial memory determination in the $Y$ Maze test. During the acquisition trial, arm 2 is closed, and the mouse moves freely between arms 1 and $3.24 \mathrm{~h}$ later, in the retrieval trial, arm 2 is opened. The mouse showed a strong preference for this novel arm, indicated by longer cumulative duration and more arm entries (AE). $\mathbf{b}$ Sham-treated mice spent significantly more time in the novel arm $(n=12 ; p<0.05$, Kruskal-Wallis test followed by Dunn's multiple comparisons test) whereas mice subjected to BCAS spent equal times in all arms, indicating spatial memory impairment ( $n=12 ; p>0.05$, Kruskal-Wallis test followed by Dunn's multiple comparisons test). c-e The distance travelled and velocity of wildtype mice subjected to sham surgery or BCAS did not differ significantly. The percentage of time spent in the surround (i. e. $\leq 5 \mathrm{~cm}$ to the wall of the arena), indicative of the level of anxiety, was also similar ( $n=12$ for each group; Mann-Whitney test) 


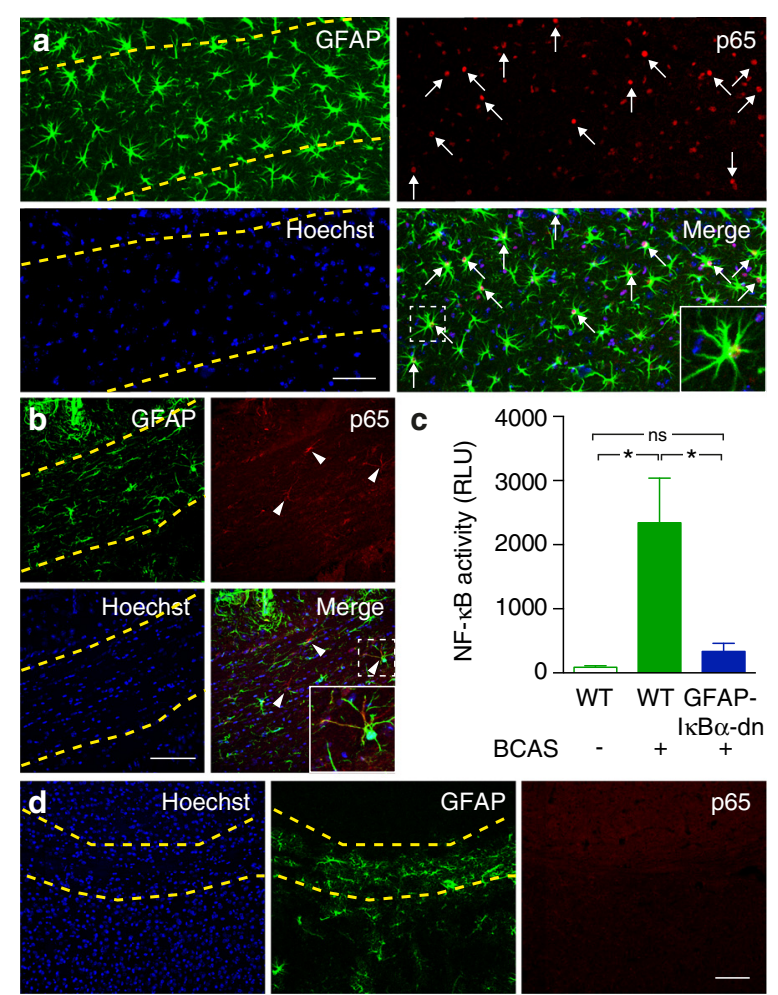

Fig. 3 Activation of the NF-kB pathway in reactive white matter astrocytes during chronic hypoperfusion. a Reactive GFAP-positive astrocytes (green) in the corpus callosum of mice subjected to BCAS are positive for the active p65 (RelA) subunit of NF-kB (red). p65 was localised to astroglial nuclei (arrows), indicated by co-localisation with the nuclear marker Hoechst 33258 (blue). b Astrocytes were rarely positive for p65 in sham-treated mice, and the signal was diffuse and localised to the cytosol (arrowheads), indicating nonactivity. c Biochemical determination of NF-kB activity in brain homogenates showed strong activation of the NF-kB pathway in wildtype mice subjected to BCAS but not in GFAP-IkBa-dn mice subjected to BCAS or sham-treated wildtype mice $(n=6$ for each group; Kruskal-Wallis test followed by Dunn's multiple comparisons test). $\mathbf{d}$ Within the corpus callosum of GFAP-IkBa-dn mice subjected to BCAS, immunohistochemistry for active $\mathrm{p} 65$ was also negative. Scale bars, $100 \mu \mathrm{m}$. Insets in A-B correspond to dashed squares and are $80 \mu \mathrm{m}$ in width. Yellow dashed lines indicate location of the corpus callosum

$0.3 \%$ of NeuN-positive neurons, respectively). Hoechstpositive and GFAP-negative punctuate p65 signals were rare $(7.3 \pm 2.4 \%$ of all Hoechst-positive p65 signals). These data indicate that the majority of white matter NF-kB activation in our model occurs in astrocytes. To quantify NF-kB activity, we used a chemiluminescent assay measuring the amount of p65 bound to the flanked DNA binding consensus sequence for NF-kB in nuclear extracts from brain homogenates. Consistent with the immunohistochemistry, this assay demonstrated strong NF-kB activation in the brains of mice subjected to BCAS compared with sham (Fig. 3c). These data indicate that reactive astrogliosis and chronic inflammation of the white matter in hypoperfused mice induces NF-kB activation in reactive astrocytes.

\section{Astrocyte-specific transgenic inhibition of NF-kB activity confers protection against structural and cognitive decline following chronic hypoperfusion}

To investigate the consequences of astroglial NF-kB activation, we used transgenic GFAP-IkB $\alpha$-dn mice in which astroglial NF-kB activation is selectively inhibited by targeting the overexpression of a dominant negative form of IkB $\alpha$ to astrocytes [14]. Earlier studies have shown that the transgene is selectively active in astrocytes, while NF-kB activity remains unchanged in neurons [14]. Following BCAS, we found that nuclear p65 was absent from reactive white matter astrocytes (Fig. 3d) and that NF-kB activity was similar to sham-treated mice (Fig. 3c).

The level and time course of cerebral blood flow reduction (Fig. 4a) and the plasticity of the posterior communicating artery (PcomA; Fig. 4b) were similar in GFAP-IkB $\alpha-\mathrm{dn}$ and wildtype mice (Laser speckle blood flow levels from each individual day of measurement were compared between GFAP-IkB $\alpha-\mathrm{dn}$ and wildtype mice; $p>0.05$ for each comparison, Mann-Whitney test). Thus, differences between these groups are unlikely to be due to variability in the degree of hypoperfusion or collateralisation between the anterior and posterior cerebral artery circulation.

Interestingly, astrogliosis was detectable in GFAP$\mathrm{I} k \mathrm{~B} \alpha-\mathrm{dn}$ mice following BCAS but reactive microglial activation and demyelination in white matter structures were significantly attenuated (Fig. 4c-e). Furthermore, axonal integrity within the corpus callosum was preserved in GFAP-IkB $\alpha$-dn mice compared with shamtreated controls (Fig. 4f). Importantly, GFAP-IkB $\alpha-\mathrm{dn}$ mice subjected to hypoperfusion spent significantly more time in the novel arm in the retrieval trial of the Y maze test (Fig. 4g), indicating preserved spatial memory function as a consequence of decreased NF-kB signaling. The open field test revealed no differences in distance moved, velocity or time spent in the surround between hypoperfused GFAP-IkB $\alpha$-dn, sham-treated GFAP-IkB $\alpha$ $\mathrm{dn}$ and wildtype mice (Fig. 4h-j). Overall, these data show that NF-kB activation in reactive astrocytes contributes to neuropathological damage and behavioural changes in our $\mathrm{VaD}$ model.

\section{Ultra high-field diffusion tensor imaging reveals preserved structural integrity of white matter tracts in GFAP-IkBa-dn mice following BCAS}

Magnetic resonance diffusion tensor imaging (DTI) is a key technology that enables the non-invasive study of structural connectivity of white matter tracts, and is an invaluable tool for the diagnosis and longitudinal study 

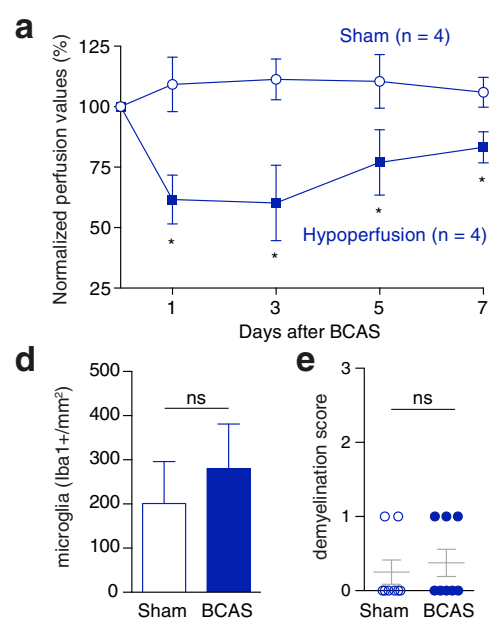

e 3

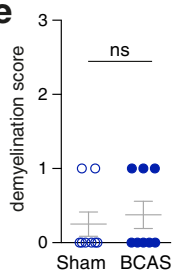

f
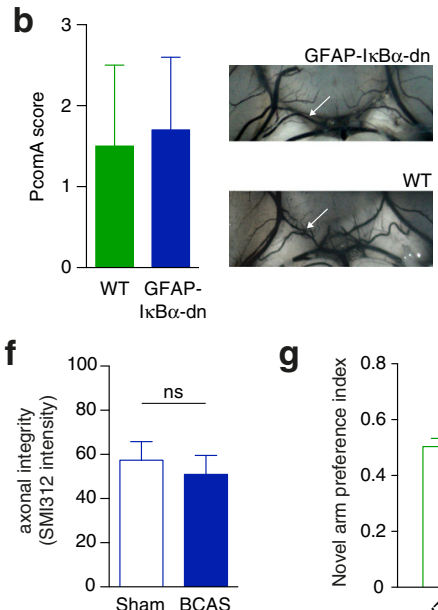

$\mathbf{C}$

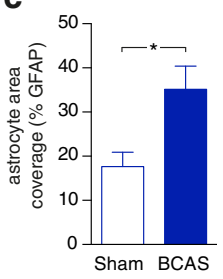

g

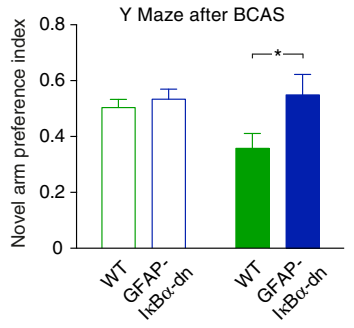

h

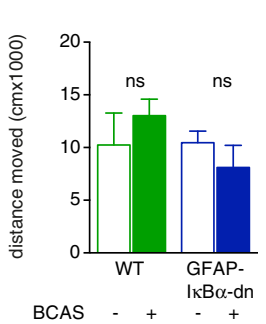

i

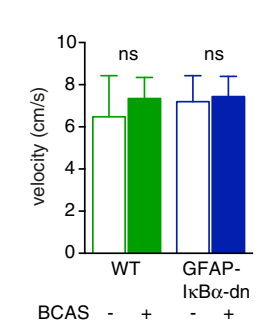

j

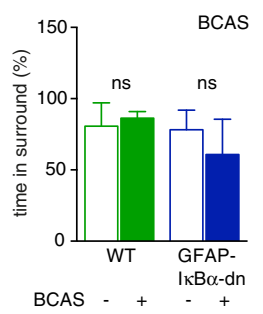

Fig. 4 GFAP-IkBa-dn mice are protected from the deleterious effects of chronic hypoperfusion. a Laser speckle contrast imaging of GFAP-IkBa-dn mice demonstrated cerebral hypoperfusion after BCAS compared with sham-treated mice $(p<0.05$, Mann-Whitney test) similar to blood flow levels observed in wildtype mice shown in Fig. 1. b India ink angiograms revealed that the patency/development of the posterior communicating artery (PComA) was similar in wildtype and GFAP-IkBa-dn mice ( $n=5$ for each group; $p>0.05$, Mann-Whitney test). $\mathbf{c}-\mathbf{f}$ Reactive astrogliosis was evident in GFAP-IkBa-dn mice after BCAS. However, microgliosis, demyelination and loss of axonal integrity were strongly ameliorated (6 weeks after BCAS; $n=6$ for each group and staining; Mann-Whitney test for each comparison). $\mathbf{g} 6$ weeks after BCAS, GFAP-|kBa-dn mice spent significantly more time in the novel arm during the retrieval trial of the $Y$ maze compared to wildtype mice subjected to BCAS, indicating preserved spatial memory function ( $n=12$ for each group; Mann-Whitney test; Two-way ANOVA: $F(1,46)=5.52, p=0.02$ for genotype effect). $\mathbf{h}$-j The distance travelled and velocity, as well as the level of anxiety, of GFAP-IkBa-dn mice subjected to sham surgery or BCAS did not differ significantly and was similar to wildtype mice. Wildtype mouse data in G-J correspond to those in Fig. 2

of patients with vascular dementia [24, 25]. Therefore, we investigated whether the preservation of axonal integrity observed in GFAP-IkB $\alpha$-dn mice was detectable by DTI MRI. DTI was performed on hypoperfused or sham-treated wildtype and GFAP-IkB $\alpha$-dn mice 6 weeks post surgery. Coronal images from brain areas encompassing the major white matter tracts (corpus callosum, internal capsule and external capsule) were acquired, and fractional anisotropy (FA), apparent diffusion coefficient (ADC), axial (AD) and radial diffusivity (RD) were measured. Representative direction-encoded colour (DEC) maps are shown in Fig. 5a. We detected significant changes in white matter FA, ADC, $\mathrm{AD}$ and $\mathrm{RD}$ values in wildtype mice subjected to BCAS compared with sham-treated mice (Fig. 5b-e and Additional file 4: Figure S4). This indicates that hypoperfusion induced deterioration in white matter integrity consistent with the observed histopathological changes. By contrast, GFAP-IkB $\alpha-d n$ mice subjected to BCAS mice did not exhibit a difference in the diffusivity measures when compared with sham-treated GFAP-IkB $\alpha$-dn mice, underlining the strong attenuation in pathology compared with wildtype animals (Fig. 5b-e). $\mathrm{T}_{1^{-}}$and $\mathrm{T}_{2}$-weighted imaging revealed no signs of overt anatomical damage in any group (Additional file 5: Figure S5).

\section{Discussion}

In this study, we investigated the role of the proinflammatory NF-kB pathway in reactive astroglia in a mouse model of vascular cognitive impairment and found that transgenic inhibition of NF-kB signaling confers protection from axonal loss, gliosis, demyelination, deterioration of white matter integrity and memory impairment.

Although vascular cognitive impairment is associated with an enormous clinical and socioeconomic burden, causal treatment options are absent, mostly because the molecular pathways triggered by chronic cerebral hypoperfusion remain poorly understood. Importantly, clinical and experimental studies have shown that vascular impairment is associated with inflammatory changes and 


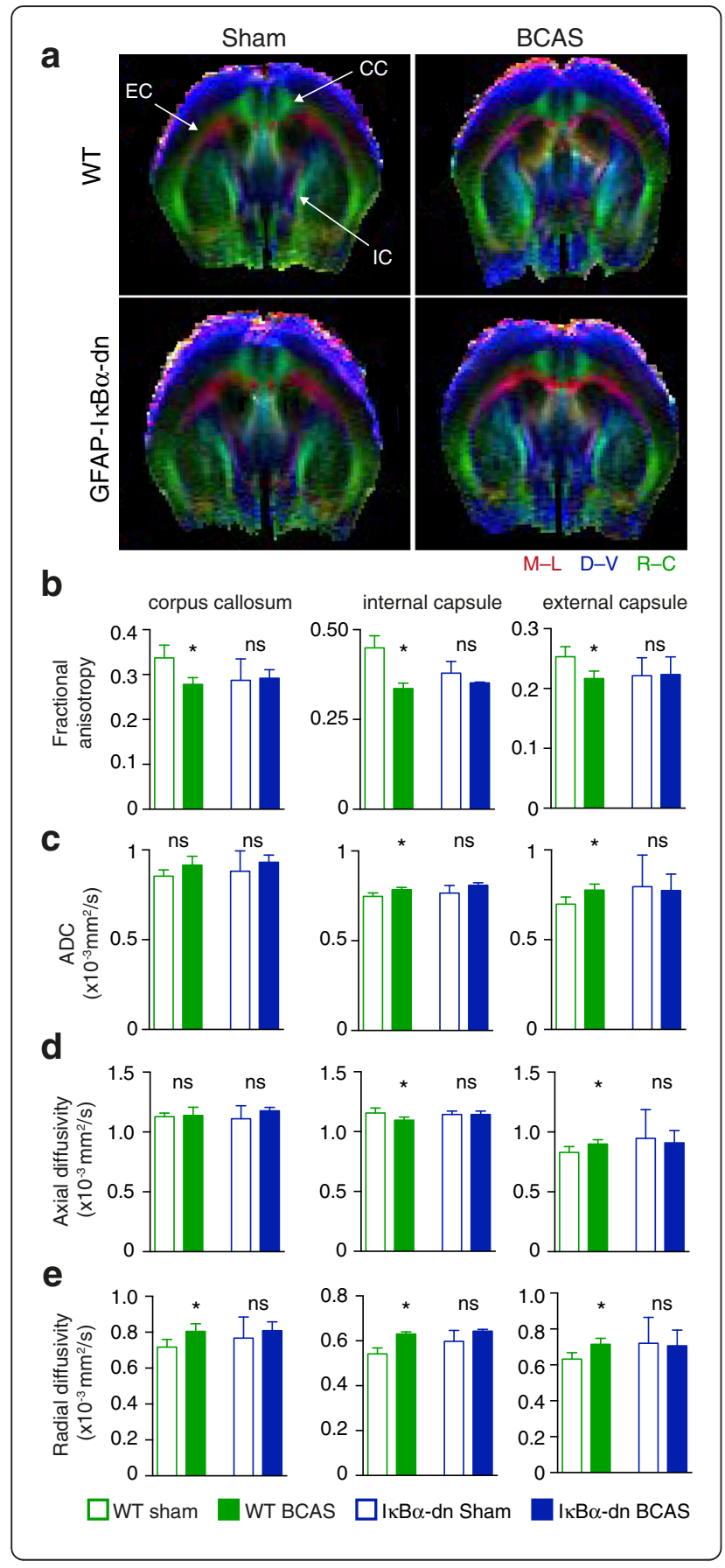

Fig. 5 Ultra high-field diffusion tensor imaging reveals preserved structural integrity of major white matter tracts in GFAP-IkBa-dn mice. a Anaesthetised wildtype or GFAP-IkBa-dn mice, either shamtreated or subjected to BCAS, were imaged using ultra high-field DTI MRI. Major white matter tracts (CC, corpus callosum; IC, internal capsule; EC, external capsule) were identified in direction-encoded colour (DEC) images (red, medial to lateral orientation; blue, dorsal to ventral orientation; green, rostral to caudal orientation) based on the primary eigenvectors. b Fractional anisotropy was significantly decreased in all regions of interest in wildtype but not in GFAP-IkBa-dn mice subjected to BCAS. c BCAS induced significant changes in the apparent diffusion coefficient (ADC) of the internal capsule and external capsule in wildtype but not in GFAP-IkBa-dn mice. d-e Axial and radial diffusivity were also significantly altered in wildtype but not in GFAP-IkBa-dn mice subjected to BCAS (wildtype sham, $n=6$; wildtype BCAS, $n=5$; GFAP-|kBa-dn sham, $n=3$; GFAP-|kBa-dn BCAS, $n=5$; Mann-Whitney test for all comparisons)

reactive gliosis of the white matter $[4-7,9]$, indicating that white matter damage is not simply a consequence of chronic oxygen deprivation but is induced and sustained by a pro-inflammatory environment. The hallmarks of chronic inflammation:reactive gliosis of white matter astrocytes and microglia, and expression of proinflammatory cytokines, were also detected in our model and associated with axonal degeneration, demyelination, spatial memory impairment and loss of white matter integrity. Importantly, the DTI signatures of hypoperfused mice corroborated with the demyelination and axonal degeneration observed on histology [26], and were comparable to those observed in clinical vascular cognitive impairment [27]. The cellular sources of IL-1 and IL-6 are difficult to define in vivo using our model. However, earlier studies have shown that microglia and, to a lesser degree, astrocytes release these cytokines during hypoxia [1, 23, 28]. Hence, both cell types may induce and sustain the pro-inflammatory environment that ultimately leads to demyelination. Moreover, progressive demyelination may further exacerbate gliosis [28].

Reactive astrogliosis is a pervasive but insufficiently understood response mechanism of the brain to acute or chronic injury [12]. Interestingly, we found that astrogliosis induces activation of the pro-inflammatory NF-kB pathway, and that transgenic inhibition of NF-kB signaling in reactive astrocytes confers protection from neurodegeneration and cognitive impairment. Notably, some degree of gliosis was evident in transgenic mice after BCAS, indicating that multiple pathways may be involved [12]. The protective effect of NF-kB on white matter integrity was also detectable using DTI, underlining the significance of DTI as a longitudinal marker of treatment response in future translational studies.

It should be noted that the model used in the present study, and arguably in previously published animal studies thus far [11], does not reflect certain aspects of clinical 
vascular cognitive impairment including the chronic evolution of the disease over decades, the predominant affection of small vessels, and cardiovascular and environmental co-morbidities. Therefore, an important question for future studies is whether the protective effect of NF-kB inhibition is evident in models that recapitulate other aspects of human vascular cognitive impairment. One other important point to address will be whether astroglial NF-kB contributes to pathways such as those mediated by hypoxia-inducible transcription factor-1 activation or perturbed glutamate uptake [29, 30] in models of vascular cognitive impairment. Furthermore, as NF-kB pathway inhibitors progress through pre-clinical development [31], it will be of great interest to investigate whether these compounds confer comparable protection from chronic hypoxia.

The NF-kB pathway has been implicated in other pathological conditions such as autoimmune or toxic demyelination and spinal cord injury [14, 23, 32]. Here, we demonstrated that activation of NF-kB in astrocytes contributes not only to white matter demyelination and axonal loss during chronic hypoxia but that an astrocytic pro-inflammatory pathway may have important consequences for cognitive outcome in neurodegenerative disease.

\section{Conclusions}

Our study has demonstrated that chronic activation and gliosis of astrocytes are central components of disease pathogenesis in a model of vascular cognitive impairment, and that therapeutic strategies targeting these pathways may lead to novel treatment options for vascular dementia.

\section{Additional file}

Additional file 1: Figure S1. Scoring system to measure demyelination. Representative LFB stainings from the corpus callosum and the corresponding grades are provided. (PDF $2173 \mathrm{~kb}$ )

Additional file 2: Figure S2. Histological and immunohistochemical changes induced by BCAS. The corpus callosum is delineated by yellow dashed lines in all images. (A) Luxol Fast Blue (LFB) staining of the corpus callosum in sham-treated mice and mice subjected to BCAS revealed fibre disarrangement, vacuoles and focal demyelination following BCAS. (B) Astrocytes, stained by an anti-GFAP antibody, became reactive, as indicated by higher area coverage, larger cell diameters, and longer processes. (C) The number of Iba1-positive microglia increased within the corpus callosum. (D) The signal intensity of axons stained with the pan-axonal marker SMI312 decreased after BCAS, indicative of axonal damage. (E) The number of NeuN-positive neuronal cell bodies was comparable in both groups. Scale bars, $100 \mu \mathrm{m}$. (PDF $7114 \mathrm{~kb}$ )

Additional file 3: Figure S3. Histological changes in the internal and external capsule. Reactive astrogliosis $(A, E)$, microgliosis $(B, F)$, axonal degeneration $(C, G)$ and demyelination $(D, H)$ in sham and BCAS animals in the internal capsule (upper row) and external capsule (lower row). (PDF 118 kb)

Additional file 4: Figure S4. Fractional anisotropy maps. Representative examples of fractional anisotropy maps in two coronal imaging planes (relative to Bregma) in wildtype or GFAP-IkBa-dn mice subjected to BCAS or sham surgery, respectively. (PDF $6689 \mathrm{~kb}$ )
Additional file 5: Figure S5. $T_{1}$ - and $T_{2}$-weighted images show no changes following BCAS. Representative examples of $T_{1}$ - and $T_{2}$-weighted coronal images in wildtype or GFAP-IkBa-dn mice subjected to BCAS or sham surgery, respectively. These images show that grey and white matter structures are devoid of pathological signal changes. (PDF $2360 \mathrm{~kb}$ )

\section{Abbreviations}

DTI, Diffusion tensor imaging; GFAP, Glial fibrillary acid protein; MRI, Magnetic resonance imaging; NF-kB, Nuclear factor kappa-light-chain-enhancer of activated B cells; VCI, Vascular cognitive impairment

\section{Acknowledgements}

We thank John Bethea (Miami) for providing the transgenic mice. We are grateful to Jan Peter and Theresa Hundt (Bonn) for help with immunohistochemistry, biochemical experiments and genotyping. We thank Marco Foddis (Berlin) for help with establishing the BCAS model, Manuel Schölling and Christoph Moehl (Bonn) for help with image analysis, and Michael Heneka (Bonn) for help with electrochemiluminescence measurements.

\section{Funding}

This work was supported by grants to GCP from the Network Of Centres Of Excellence In Neurodegeneration (CoEN), EU Joint Programme-Neurodegenerative Disease Research (JPND) program (EU Horizon 2020 research and innovation program, grant agreement No 643417/DACAPO-AD), and the DZNE.

\section{Availability of data and material}

The paper does not include proprietary material or any data for which deposition in a public repository is mandatory, but all relevant data can be made available from the corresponding author upon request.

\section{Authors' contributions}

RS, TS and GCP implemented the methodology and designed the experiments. RS developed and optimised the MRI sequences. RS, TS, FG, CR, KT and AD performed the experiments. RS, TS, FG and GCP analysed the data. RS and GCP wrote the manuscript. All authors discussed the results and commented on the manuscript at all stages. All authors read and approved the final manuscript.

\section{Competing interests}

The authors declare that they have no competing interests.

\section{Consent for publication}

Not applicable.

Ethics approval and consent to participate

All experiments were performed according to animal care guidelines and approved by a local animal welfare committee.

Received: 15 July 2016 Accepted: 19 July 2016

Published online: 04 August 2016

\section{References}

1. Iadecola C. The Pathobiology of Vascular Dementia. Neuron. 2013;80:844-66

2. ladecola C. Neurovascular regulation in the normal brain and in Alzheimer's disease. Nat Rev Neurosci. 2004;5:347-60.

3. Gorelick PB, Scuteri A, Black SE, Decarli C, Greenberg SM, ladecola C, et al. Vascular contributions to cognitive impairment and dementia: a statement for healthcare professionals from the american heart association/american stroke association. Stroke. 2011;42:2672-713.

4. Rouhl RP, Damoiseaux JG, Lodder J, Theunissen RO, Knottnerus IL, Staals J, et al. Vascular inflammation in cerebral small vessel disease. Neurobiol Aging. 2012;33:1800-6.

5. Aribisala BS, Wiseman S, Morris Z, Valdés-Hernández MC, Royle NA, Maniega SM, et al. Circulating inflammatory markers are associated with magnetic resonance imaging-visible perivascular spaces but not directly with white matter hyperintensities. Stroke. 2014:45:605-7.

6. Back SA, Kroenke CD, Sherman LS, Lawrence G, Gong X, Taber EN, et al. White matter lesions defined by diffusion tensor imaging in older adults. Ann Neurol. 2011;70:465-76. 
7. Juma WM, Lira A, Marzuk A, Marzuk Z, Hakim AM, Thompson CS. C-reactive protein expression in a rodent model of chronic cerebral hypoperfusion. Brain Res. 2011;1414:85-93.

8. Wardlaw JM, Doubal FN, Valdes-Hernandez M, Wang X, Chappell FM, Shuler $\mathrm{K}$, et al. Blood-brain barrier permeability and long-term clinical and imaging outcomes in cerebral small vessel disease. Stroke. 2013;44:525-7.

9. Rosenberg GA, Bjerke M, Wallin A. Multimodal markers of inflammation in the subcortical ischemic vascular disease type of vascular cognitive impairment. Stroke. 2014;45:1531-8.

10. Akiguchi I, Tomimoto H, Suenaga T, Wakita H, Budka H. Alterations in glia and axons in the brains of Binswanger's disease patients. Stroke. 1997;28:1423-9.

11. Hainsworth AH, Markus HS. Do in vivo experimental models reflect human cerebral small vessel disease? A systematic review. J Cereb Blood Flow Metab. 2008;28:1877-91.

12. Burda JE, Sofroniew MV. Reactive Gliosis and the Multicellular Response to CNS Damage and Disease. Neuron. 2014;81:229-48.

13. Shibata M, Ohtani $\mathrm{R}$, Ihara $\mathrm{M}$, Tomimoto $\mathrm{H}$. White matter lesions and glial activation in a novel mouse model of chronic cerebral hypoperfusion. Stroke. 2004;35:2598-603.

14. Brambilla R, Bracchi-Ricard V, Hu W-H, Frydel B, Bramwell A, Karmally S, et al. Inhibition of astroglial nuclear factor kappaB reduces inflammation and improves functional recovery after spinal cord injury. J Exp Med. 2005;202: $145-56$.

15. Reimer MM, McQueen J, Searcy L, Scullion G, Zonta B, Desmazieres A, et al. Rapid Disruption of Axon-Glial Integrity in Response to Mild Cerebral Hypoperfusion. J Neurosci. 2011;31:18185-94.

16. Coltman R, Spain A, Tsenkina Y, Fowler JH, Smith J, Scullion G, et al, Selective white matter pathology induces a specific impairment in spatial working memory. Neurobiol Aging. 2011;32:2324. e7-e12.

17. Miki K, Ishibashi S, Sun L, Xu H, Ohashi W, Kuroiwa T, et al. Intensity of chronic cerebral hypoperfusion determines white/gray matter injury and cognitive/motor dysfunction in mice. J Neurosci Res. 2009;87:1270-81.

18. Bracchi-Ricard V, Brambilla R, Levenson J, Hu W-H, Bramwell A, Sweatt JD, et al. Astroglial nuclear factor-kB regulates learning and memory and synaptic plasticity in female mice. J Neurochem. 2008:104:611-23.

19. Park L, Zhou P, Pitstick R, Capone C, Anrather J, Norris EH, et al. Nox2derived radicals contribute to neurovascular and behavioral dysfunction in mice overexpressing the amyloid precursor protein. Proc Natl Acad Sci U S A. 2008;105:1347-52.

20. Paxinos G, Franklin KBJ. The Mouse Brain in Stereotaxic Coordinates. 3rd ed. London: Academic; 2008.

21. Kaltschmidt B, Widera D, Kaltschmidt C. Signaling via NF-kappaB in the nervous system. Biochim Biophys Acta. 2005;1745:287-99.

22. Hayden MS, Ghosh S. Shared principles in NF-kappaB signaling. Cell. 2008; 132:344-62.

23. Raasch J, Zeller N, van Loo G, Merkler D, Mildner A, Erny D, et al. IkB kinase 2 determines oligodendrocyte loss by non-cell-autonomous activation of NF-kB in the central nervous system. Brain. 2011;134:1184-98.

24. Patel B, Markus HS. Magnetic resonance imaging in cerebral small vessel disease and its use as a surrogate disease marker. Int J Stroke. 2011:6:47-59.

25. Wardlaw JM, Smith C, Dichgans M. Mechanisms of sporadic cerebral small vessel disease: insights from neuroimaging. Lancet Neurol. 2013;12:483-97.

26. Zhang J, Aggarwal M, Mori S. Structural insights into the rodent CNS via diffusion tensor imaging. Trends Neurosci. 2012;35:412-21.

27. Nitkunan A, Barrick TR, Charlton RA, Clark CA, Markus HS. Multimodal MRI in cerebral small vessel disease: its relationship with cognition and sensitivity to change over time. Stroke. 2008;39:1999-2005.

28. Hanisch UK. Microglia as a source and target of cytokines. Glia. 2002;40:140-55.

29. Rius J, Guma M, Schachtrup C, Akassoglou K, Zinkernagel AS, Nizet V, et al. NF-kappaB links innate immunity to the hypoxic response through transcriptional regulation of HIF-1alpha. Nature. 2008;453:807-11.

30. Dallas M, Boycott HE, Atkinson L, Miller A, Boyle JP, Pearson HA, et al. Hypoxia suppresses glutamate transport in astrocytes. J Neurosci. 2007;27:3946-55.

31. Miller SC, Huang R, Sakamuru S, Shukla SJ, Attene-Ramos MS, Shinn P, et al. Identification of known drugs that act as inhibitors of NF-kB signaling and their mechanism of action. Biochem Pharmacol. 2010;79:1272-80.

32. van Loo G, de Lorenzi R, Schmidt H, Huth M, Mildner A, Schmidt-Supprian $M$, et al. Inhibition of transcription factor NF-kappaB in the central nervous system ameliorates autoimmune encephalomyelitis in mice. Nat Immunol. 2006;:954-61

\section{Submit your next manuscript to BioMed Central and we will help you at every step:}

- We accept pre-submission inquiries

- Our selector tool helps you to find the most relevant journal

- We provide round the clock customer support

- Convenient online submission

- Thorough peer review

- Inclusion in PubMed and all major indexing services

- Maximum visibility for your research

Submit your manuscript at www.biomedcentral.com/submit
Biomed Central 